\title{
A public health response to a newly diagnosed case of hepatitis $C$ associated with lapse in Infection Prevention and Control practices in a dental setting in Ontario, Canada
}

\author{
Cassandra Johnston ${ }^{1 *}$, Vidya Sunil ${ }^{1}$, Dorothea Service ${ }^{1}$, Anne Marie Holt ${ }^{1}$, Gary Garber ${ }^{2,3}$, \\ Liane Macdonald ${ }^{2,4}$, Erik Kristjanson², Tony Mazzulli2,4,5, Romy Olsha², David Ryding², \\ Avis Lynn Noseworthy ${ }^{1}$
}

\begin{abstract}
Background: Haliburton, Kawartha, Pine Ridge District Health Unit (HKPRDHU) investigated an exposure in an Ontario operatory dental facility related to a newly diagnosed hepatitis $C$ virus $(\mathrm{HCV})$ infection caused by a virus with an uncommon hepatitis $\mathrm{C}$ genotype. Lapses in Infection Prevention and Control (IPAC) and a second epidemiologically-linked case (with the same uncommon hepatitis $C$ genotype) were identified, prompting a broader public health response and outbreak investigation.
\end{abstract}

Objectives: a) To describe the investigation of a newly diagnosed case of hepatitis $C$; b) to describe the broader public health response, and c) to address a paucity in the literature related to the risk of disease transmission in dental settings due to IPAC lapses.

Methods: A collaborative approach with two dental practices, public health partners and regulatory bodies was used. An IPAC inspection was completed to determine and mitigate the risk of blood borne infection transmission within the facilities. Appropriate protocols were followed for the IPAC investigation and public health response.

Results: The investigation identified a risk of potential HCV transmission between two cases linked to the same dental facility. There were no other epi-linked cases of HCV identified. Challenges included a lack of adherence to IPAC standards in one of the dental settings and awareness in the dental community regarding $\mathrm{HCV}$ transmission, coordination with regulatory bodies and public health experts and low uptake of laboratory testing by patients.

Conclusion: Despite the unique challenges associated with the investigation, HKPRDHU conducted a successful IPAC lapse investigation and public health response. Public health units need to maintain collaborative approaches with regulated health professionals, their regulatory bodies and public health experts.
This work is licensed under a Creative Commons Attribution 4.0 International License.

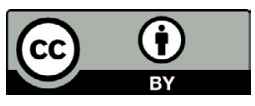

Affiliations

${ }^{1}$ Haliburton, Kawartha, Pine Ridge District Health Unit, Port Hope, ON

2 Public Health Ontario, Toronto, $\mathrm{ON}$

${ }^{3}$ Ottawa Hospital Research Institute, Department of Medicine, University of Ottawa, Ottawa, ON

${ }^{4}$ Dalla Lana School of Public Health, University of Toronto, Toronto, ON

${ }^{5}$ Mount Sinai Hospital, University Health Network, Toronto, ON

*Correspondence:

cjohnston@hkpr.on.ca

Suggested citation: Johnston C, Sunil V, Service D, Holt AM, Garber G, Macdonald L, Kristjanson E, Mazzulli T, Olsha R, Ryding D, Noseworthy AL. A public health response to a newly diagnosed case of hepatitis $C$ associated with lapse in Infection Prevention and Control practices in a dental setting in Ontario, Canada. Can Commun Dis Rep 2021;47(7/8):347-52. https://doi.org/10.14745/ccdr.v47i78a08

Keywords: IPAC, hepatitis C, dental, lapse, transmission

\section{Introduction}

The Haliburton, Kawartha, Pine Ridge District Health Unit (HKPRDHU) was notified through routine reporting of infectious diseases of a newly diagnosed case of hepatitis $\mathrm{C}$ virus (HCV) infection, genotype 2, in a client who had attended two different dental clinics (Facility A and Facility B) during the viral incubation period. The index case had no other reported current or past risk 
factors related to HCV infection. The index case was defined as the newly confirmed case for this suspect outbreak investigation. An Infection Prevention and Control (IPAC) investigation was launched to determine the risk of HCV transmission in both dental settings. An HCV outbreak was not declared but this investigation was conducted as a potential outbreak. The objectives of this article are to 1) describe the investigation of a newly diagnosed case of HCV in a client who had potential exposures at two different community dental clinics; 2) describe the broader public health response; and 3) address a paucity in the literature related to the risk of disease transmission in dental clinics due to IPAC lapses.

\section{Background}

Hepatitis $C$ virus infection is a reportable disease of public health significance (1). Dental standards of practice and IPAC best practices support the prevention of blood borne infection (BBI) transmission (2). Hepatitis $C$ virus infection-related IPAC investigations in dental facilities associated with improper IPAC practices have occasionally been reported $(3,4)$. Public health units in Ontario are required to investigate IPAC complaints and suspect IPAC lapses associated with an infectious disease transmission risk, adhering to infectious disease investigation principles and protocols (5).

Hepatitis $C$ virus is a ribonucleic acid (RNA) virus belonging to the Flaviviridae family and has at least six major genotypes and approximately 100 subtypes. Genotype 1 is the predominant genotype in Canada (6), whereas genotype 2 accounts for approximately $10 \%-15 \%$ of Canadian HCV infections (7-9).

Reported case counts and rates of HCV in Ontario have increased in recent years. The newly reported rate of $\mathrm{HCV}$ in Ontario was 36.5 cases per 100,000 population in 2018 , of which $22.5 \%$ were newly acquired infections (10). Hepatitis $C$ virus is primarily transmitted by blood-to-blood contact and acute infection is often asymptomatic.

\section{Methods}

Review of the manuscript by privacy experts at HKPRDHU and Public Health Ontario (PHO) was performed.

The index case was diagnosed with HCV in December of year 0 and was reported to HKPRDHU in January of year 1. The HKPRDHU began an IPAC investigation in January of year 1 to determine if either of the two clinics attended by the HCV-positive client (Facility A or Facility B) could have been the source of HCV transmission. The index case received procedures at both facilities within the incubation period for HCV. Dental procedures included teeth cleaning at Facility A and, subsequently, a tooth extraction with intravenous medication at Facility B.
An IPAC inspection was completed at each facility to determine and mitigate the risk of $\mathrm{BBI}$ transmission within the facilities. Appropriate protocols $(5,6,11)$ were followed for the IPAC investigation and public health response.

\section{Infection Practices and Control inspections}

Onsite inspections were conducted for both facilities to determine if there was evidence of $\mathrm{BBI}$ transmission risk through IPAC lapses. The inspection team included a public health inspector, a nurse certified in infection control and a dental hygienist. Other public health professionals assisted in inspections and visits with the facilities as needed. The inspection team utilized standardized $\mathrm{PHO}$ checklists $(12,13)$ to guide the inspections. Applicable regulatory bodies were contacted by the investigation team prior to conducting inspections for any support required and were invited to participate.

\section{Case definitions}

An HCV outbreak is defined as the occurrence of two or more cases of HCV infection linked by time or a common exposure source or setting (6). The preliminary case definition in this investigation included both settings; however, the final case definition for the investigation was as follows: a laboratory-confirmed case of HCV genotype 2 who had dental procedures on or between November 10, year 0 to November 20, year 0 (three business days before, on the day of, or three business days after the day of the procedure of the index case at Facility B).

\section{Case-finding}

The index case (female, 50-60 years of age) had her dental procedure completed on November 15, year 0 and became symptomatic with acute HCV infection on December 9, year 0 . Blood work detected elevated liver enzymes and HCV antibodies, with further blood work indicating detection of $\mathrm{HCV}$ RNA and genotype 2 HCV.

As a preliminary case-finding step in the investigation, an $\mathrm{HCV}$ case look-back exercise was conducted using the Integrated Public Health Information System (iPHIS) database to identify any confirmed cases of HCV reported January to December year 0 in the HKPRDHU's jurisdiction who had dental procedures at one of the clinics reported in the investigation, or any case who had identified a dental procedure as a risk factor. None were reported as being associated with Facility A or Facility B.

Patient rosters were collected from both dental facilities. The first roster was received from Facility B in April year 1. Case lookback regarding the patients listed was conducted by $\mathrm{PHO}$ using laboratory information system-based data. The identification of a second case (female, 70-80 years of age), previously positive (defined as any case reported historically to public health) in year 0 minus 10, with chronic HCV (genotype 2), prompted the continuation of case look-back at Facility $B$. This case was seen at Facility $B$ on the same day and prior to the index case. In May year 1, a patient notification was initiated, to identify any new 
$\mathrm{HCV}$ cases associated with this investigation. The notification was sent to patients seen at Facility B between three business days prior to the index case's procedure (November 10, year 0 ) and up until IPAC practices met standards (February 21, year 1).

\section{Chart review}

Following the second IPAC inspection of Facility B, a chart review was conducted for all patient procedures that took place on the day the index case and potential source case were seen. On the day of suspected transmission, chart documentation confirmed a single operatory room was used for all procedures completed on that day and overlapping appointments were scheduled and occurred (Figure 1).

Figure 1: Dental procedure start and end time ${ }^{a}$ of the procedure at the dental Facility $\mathrm{B}^{\mathrm{b}}$

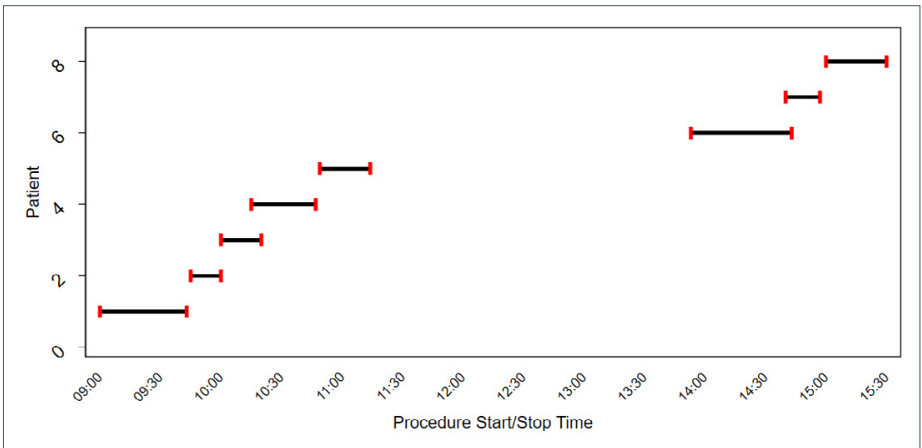

One appointment is missing from Figure 1 due to incomplete documentation related to an appointment that began at 13:00 hours but no end time had been documented

This figure shows the procedure start and end time for each patient seen on the day both potential source case and index case underwent their dental procedure at Facility $B$ in year 0 , the day potential transmission may have happened

\section{Public health response}

Patients who had dental procedures conducted at Facility $B$ between November 10, year 0 and February 21, year 1 were notified in writing of the potential risk of exposure to $\mathrm{HCV}$, and were advised to get tested for HCV, hepatitis $B$ and human immunodeficiency virus (HIV). A total of 264 initial notification letters signed by the Medical Officer of Health and the Dental Surgeon along with a blood test requisition were mailed out on May 25, year 1. For those patients who required a follow-up test, 167 additional notification letters were sent recommending repeat testing six months after the date of the patient's dental appointment. Phone calls were made to follow-up with each patient to complete an assessment and to provide health education using the Health Canada HCV fact sheet (14). A media release was also issued in consultation with the owner of Facility $B$, and an information page was created on the HKPRDHU's website.

\section{Epidemiologic and statistical analyses}

Laboratory results received were entered into an Excel database and were exported into Stata 15 (15) for further analysis to generate an epidemiological curve and summary.
An Excel spreadsheet was used for tracking patient follow-up as well as to log any community calls.

\section{Laboratory investigation}

Patient blood samples were requested to be sent to the $\mathrm{PHO}$ laboratory for testing. All serum specimens for HCV antibody were initially tested using the Abbott ARCHITECT anti-HCV antibody test. Negative results were reported with no further testing performed. Positive or indeterminate results underwent supplemental testing using a second validated assay (Siemens AD VIA Centaur HCV Assay or the Ortho Clinical Diagnostics VITROS HCV assay), and final HCV antibody results were based on the results of both tests. The HCV genotyping/subtyping were performed on all first-time HCV RNA-positive specimens with a viral load of $500 \mathrm{IU} / \mathrm{mL}$ or greater.

\section{Results}

Investigation findings ruled out Facility $A$ as a possible source of the HCV infection. Investigation findings in Facility B supported proceeding with a case-finding exercise, including patient notification, to identify any possible epidemiologically linked cases related to the facility.

Considering the person, place and time perspectives, the same uncommon genotype identified; the second case identified had a high likelihood of being the source case. Unfortunately, further confirmatory subtype testing of the probable source case could not be conducted due to the demise of the case. The demise was unrelated to Facility B.

\section{Infection Prevention and Control investigation of the facilities}

Inspections were conducted focusing on reprocessing of dental and medical equipment. No evidence of a lapse in reprocessing practices supporting $\mathrm{BBI}$ transmission risk was observed during the inspection at Facility $A$. The inspection at Facility $B$ identified issues with reprocessing practices that may have led to an IPAC lapse. A risk assessment was conducted in collaboration with $\mathrm{PHO}$, which prompted a second inspection of Facility $\mathrm{B}$ to observe IPAC practices in relation to patient procedures, including medication administration practices.

During the initial inspection of Facility $B$, key issues identified included the following:

- Lack of physical separation between the dirty and clean areas for reprocessing

- Reassembly and over packaging of instruments prior to sterilization

- Inconsistent use of chemical indicators with every wrapped pack/pouch

- Routine non-availability of biological indicator results 
- Release of instruments prior to completion of sterilization process

- No evidence of monitoring the physical parameters of the sterilizer for each cycle

- Incomplete record keeping

- Use of damaged instruments

During the second inspection of Facility $B$, key issues identified included the following:

- Medications were pre-drawn and not labelled for specific patients

- Inadequate use of personal protective equipment by staff during patient procedures

- Inconsistent pre-cleaning of dirty dental handpieces prior to sterilization

- Improper use of contaminated unpunctured carpules: unpunctured carpules were taken from a contaminated instrument tray and were used for a different patient (the carpules were "reprocessed" with a disinfectant wipe prior to use with a different patient)

- Retention of unused medication syringes from one procedure were subsequently used for additional procedures on other patients

- No staff dedicated to overseeing IPAC in the facility

The Health Unit issued the owner of Facility B orders to correct the IPAC deficiencies identified during the inspections, resulting in a temporary closure of Facility B. Follow-up inspections were completed by the Health Unit in collaboration with the dental regulatory body and all deficiencies were found to have been corrected.

\section{Descriptive data analyses}

Descriptive analyses of the initial and 6-month follow-up lab results were completed.

Figure 2 shows the number of laboratory results for HCV received for individuals who received dental procedures (by treatment date) at Facility B between November 10, year 0 and February 21, year 1. Out of the 264 patients notified, 259 required testing. The five patients from the patient roster who received only a dental consult did not require testing. Of the 259 who required testing, 231 completed the initial test (89.2\%). Among the initial test results there were two previously positive $\mathrm{HCV}$ cases, in addition to the probable source case and index case. With the exception of the index case, three of these four cases had other risk factors for HCV infection. Twenty-eight patients did not have an initial lab test as recommended by the Health Unit.

Figure 3 shows the test results for patients (among the same patient cohort) for whom a 6-month follow-up HCV laboratory testing was required. The 6-month testing was not required for all patients: among the 145 notified patients, 99 completed their 6-month HCV laboratory test (68.2\%). There were no positive test results.

Figure 2: Number of positive and negative hepatitis $C$ laboratory testing results, by date of dental procedure, at dental Facility $B$ at the time of the initial investigation

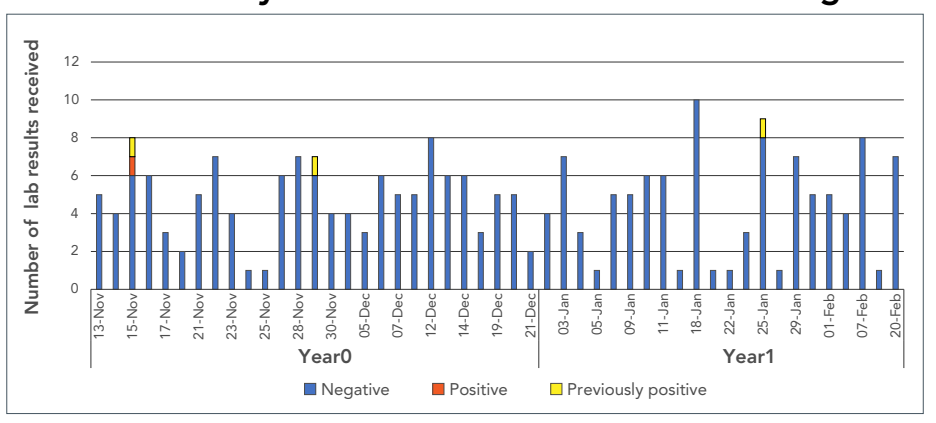

Figure 3: Number of positive and negative hepatitis $C$ laboratory results at the 6-month follow-up, by date of dental procedure, at dental Facility $B$

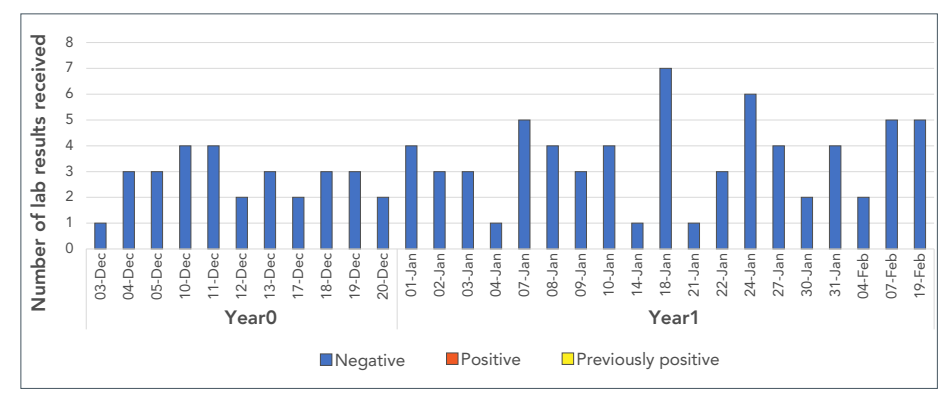

\section{Case management and public health resource allocation}

Approximately 805 interventions (phone calls, faxes and email interactions) were conducted by public health nurses in the process of patient notification and related follow-up. An average of three interventions per patient was conducted (ranging from one to seven). The total staffing hours spent on HKPRDHU's response to this suspected outbreak was $1,187.5$ hours.

\section{Discussion}

Our IPAC investigation led to a broader public health response. To our knowledge, this is the first time in Ontario that an IPAC investigation in a dental office was initiated based on a reported confirmed case of HCV with an uncommon genotype.

No evidence was found to suggest that Facility A was the source of the HCV infection. Through a collaborative consultation process, it was determined that a case look-back exercise would be completed for three days before, the day of, and three days after the index case was seen at Facility B. Based on the viability 
of HCV on surfaces (16), and literature on HCV transmission through medication administration (17), a narrow search window of a few days around the index case's likely exposure was deemed appropriate.

Through our patient notification process, other than the probable source case and the epidemiologically-linked index case, no further related cases were identified. Although we were not able to confirm further transmission of HCV beyond the index case, the epidemiological and IPAC investigation findings provided us with enough evidence to support our hypothesis that HCV transmission may have occurred at Facility B between the source case and the index case.

At the time of this investigation, there was a perceived lack of awareness among the dental community regarding the potential for $\mathrm{HCV}$ transmission related to dental procedures. However, concern about the possible spread of BBls and other diseases is growing (2), and our findings suggest that lapses in IPAC practices could result in the transmission of HCV. Further studies and publications of relevant investigations are needed to clearly understand the implications of HCV transmission during dental procedures, both due to direct transmission and through virus survival on surfaces.

Results from this IPAC investigation suggest that the lack of designated IPAC staff within Facility B led to inadequate IPAC procedures. One staff person assisting with dental procedures was also responsible for equipment reprocessing throughout the day, and Figure 1 shows how limited time there was between appointments. Guidelines recommend having a staff person designated to manage IPAC in each office (18), and reprocessing requires specific training and skills along with adequate time and staffing.

Issues discovered during the subsequent inspection of Facility B related to improper medication practices significantly increased the likelihood of BBI transmission $(2,3)$. More emphasis on IPAC requirements for dental facilities is recommended to ensure community dental facilities are meeting IPAC standards. It is recommended that health units and regulatory bodies seek out opportunities to conduct proactive inspections and provide ongoing education and support to dental health professionals regarding IPAC practices $(19,20)$, with emphasis on reprocessing and safe medication practices.

The development of best practice guidelines for IPAC inspections and investigations should help to support Ontario health units to streamline their approaches to IPAC investigations.

\section{Limitations}

Despite extensive patient follow-up, obtaining complete lab test results for all exposed patients through public notification was an identified challenge. While our initial test response rate was $89.2 \%$, our 6-month follow-up test response rate dropped to $68.2 \%$.
Previous studies have shown that HVC infection related to dental settings is rarely reported in the scientific literature (21). There is minimal scientific evidence of transmission of $\mathrm{HCV}$ in dental settings, which posed challenges to this investigation. This could be complicated by the fact that many newly infected individuals are asymptomatic, and if diagnosed subsequently, they cannot link their infection to the procedure.

\section{Conclusion}

The HKPRDHU supported the IPAC investigation and broader public health response related to a dental facility, with an epidemiologically-linked pair of HCV cases with an uncommon genotype. The public health measures included case follow-up, patient notification, communication of test results, provision of counseling and resources for patients and the public, recommendation of IPAC measures and collaboration with healthcare professionals. This investigation presented the Health Unit with unique challenges as it was complex, with multiple regulatory bodies involved. Community dental facilities need to be informed of the most current IPAC requirements to prevent risk of transmission of BBls. Public health efforts will continue to focus on collaborating with and supporting our dental health professionals to mitigate any such risks to the public.

\section{Authors' statement}

CJ, VS, and DS - Conceived the analysis, analyzed the data, and drafted the manuscript

$\mathrm{AMH}$ and $\mathrm{ALN}$ - Contributed to the content of the manuscript and interpreted the data

GG, LM and DR - Reviewed the manuscript

EK, TM and RO - Contributed to laboratory content of the manuscript

All authors approved the final version to be published and agreed to be accountable for all aspects of the work.

The content and view expressed in this article are those of the authors and do not necessarily reflect those of the Government of Canada.

\section{Competing interests}

None.

\section{Acknowledgements}

We would like to acknowledge all Haliburton, Kawartha, Pine Ridge District Health Unit (HKPRDHU) staff who assisted with the investigation, including case follow-up and contact tracing, Public Health Ontario laboratory for conducting laboratory diagnosis, and case look-back. We also thank our dental facilities who collaboratively worked with the Health Unit on this investigation.

\section{Funding}

There was no funding received for the completion of this paper. 


\section{References}

1. Government of Ontario. Health Protection and Promotion Act, R.S.O. 1990, c. H. 7. Government of Ontario; 1990, (updated 2018; accessed 2019-06-27). https://www.ontario. ca/laws/statute/90h07

2. Royal College of Dental Surgeons of Ontario. Standard of Practice: Infection Prevention and Control in The Dental Office. Toronto (ON): RCDSO; 2018 (accessed 2019-05-23). https://az184419.vo.msecnd.net/rcdso/pdf/standards-ofpractice/RCDSO_Standard_of_Practice_IPAC.pdf

3. Weaver JM. Confirmed transmission of hepatitis $C$ in an oral surgery office. Anesth Prog 2014;61(3):93-4. DOI PubMed

4. Simcoe Muskoka District Health Unit. Infection Prevention and Control Lapse SMH; 2017 (update 2018-06-18; accessed 2019-05-01). http://www.simcoemuskokahealth. org/Topics/InfectiousDiseases/InfectionPrevention/ Investreports/Infection-Prevention-and-Control-LapseReport-for-Joe-Philip-and-Associates/Infection-Preventionand-Control-Lapse-update-June-19-2018

5. Ministry of Health and Long-Term Care. Infection Prevention and Control Complaint Protocol. MHLTC; 2018 (accessed 2019-05-23). http://www.health.gov.on.ca/en/pro/programs/ publichealth/oph_standards/docs/protocols_guidelines/ IPAC_Complaint_Protocol_2018_en.pdf

6. Ministry of Health and Long-Term Care. Infectious Diseases Protocol: Appendix A Disease-Specific Chapters: Hepatitis C. MHLTC; 2018 (accessed 2019-10-31). http://www.health. gov.on.ca/en/pro/programs/publichealth/oph_standards/ docs/hep_c_chapter.pdf

7. Antonishyn NA, Ast VM, McDonald RR, Chaudhary RK, Lin L, Andonov AP, Horsman GB. Rapid genotyping of hepatitis $C$ virus by primer-specific extension analysis. J Clin Microbiol 2005;43(10):5158-63. DOI PubMed

8. Marotta P, Cooper CL, Wong DK, Farley J, Elkashab M, Peltekian KM, Abadir N, Woolstencroft RN, Bailey RJ. Impact of advanced fibrosis and cirrhosis on sustained virologic response of HCV G1-infected patients: Results of the Canadian power program (Poster presentation). 58 ${ }^{\text {th }}$ annual meeting of the American Association for the Study of Liver Diseases, 31 October-4 November 2008. San Francisco, California, USA. http://www.hivandhepatitis.com/ legacysite/2008icr/aasld/posters/SCI080887-01POWER_ FINAL.pdf

9. Chaudhary R, Tepper M, Eisaadany S, Gully PR. Distribution of hepatitis $C$ virus genotypes in Canada: Results from the LCDC Sentinel Health Unit Surveillance System. Can J Infect Dis 1999;10(1):53-6. DOI PubMed

10. Public Health Ontario. Hepatitis C in Ontario, 2018: Surveillance summary one year after a case definition update. Toronto (ON): PHO; 2020 (accessed 2020-09-30). https://www.publichealthontario.ca/-/media/ documents/r/2020/report-hepc-surveillance-2018.pdf?la=en
11. Ontario Ministry of Health and Long-Term Care. Infection Prevention and Control Disclosure Protocol. MHLTC: 2018 (accessed 2019-05-23). http://www.health.gov.on.ca/en/ pro/programs/publichealth/oph_standards/docs/protocols_ guidelines/Infection_Prevention_and_Control_Disclosure_ Protocol_2018_en.pdf

12. Public Health Ontario. IPAC Checklist for Dental Practice: Core Elements. PHO; 2018 (updated 2019). https://www. publichealthontario.ca/-/media/documents/C/2019/ checklist-ipac-dental-core.pdf?la=en

13. Public Health Ontario. IPAC Checklist for Dental Practice: Reprocessing of Dental/Medical Equipment/Devices. PHO; 2018 (updated 2019). https://www.publichealthontario. $\mathrm{ca} /-/$ media/documents/C/2019/checklist-ipac-dentalreprocessing.pdf?la=en

14. Government of Canada. Hepatitis C. Government of Canada; 2019 (accessed 2019-06-27). https://www.canada. ca/en/public-health/services/diseases/hepatitis-c.html

15. StataCorp. (2017). Stata Statistical Software: Release 15. College Station, TX: StataCorp LLC.

16. Canadian Center for Occupational Health and Safety. OSH Answers Fact Sheets: Hepatitis C. CCOHS; 2020 (accessed 2020-09-20). https://www.ccohs.ca/oshanswers/ diseases/hepatitis_c.html

17. Schaefer MK, Perkins KM, Perz JF. Patient notification events due to syringe reuse and mishandling of injectable medications by health care personnel-United States, 2012-2018: summary and recommended actions for prevention and response. Mayo Clin Proc 2020;95(2):243-54. DOI PubMed

18. College of Dental Hygienists of Ontario. Infection Prevention and Control (IPAC) Guidelines. CDHO; 2019 (accessed 2019-12-10). http://www.cdho.org/docs/defaultsource/pdfs/reference/guidelines/cdho-ipac-guidelines.pdf

19. Willmore J, Ellis E, Etches V, Labrecque L, Osiowy C, Andonov A, McDermaid C, Majury A, Achonu C, Maher M, MacLean B, Levy I. Public health response to a large-scale endoscopy infection control lapse in a nonhospital clinic. Can J Infect Dis Med Microbiol 2015;26(2):77-84. DOI PubMed

20. Cadieux G, Bhatnagar A, Schindeler T, Prematunge C, Perron D, Willmore J. Assessment of the infection prevention and control learning needs of Ottawa community-based healthcare providers. Can J of Infect Cont. 2019;34(3):135-40. https://ipac-canada. org/photos/custom/CJIC/IPAC_Fall2019_Cadieux.pdf

21. Cleveland JL, Gray SK, Harte JA, Robison VA, Moorman AC, Gooch BF. Transmission of blood-borne pathogens in US dental health care settings: 2016 update. J Am Dent Assoc 2016;147(9):729-38. DOI PubMed 